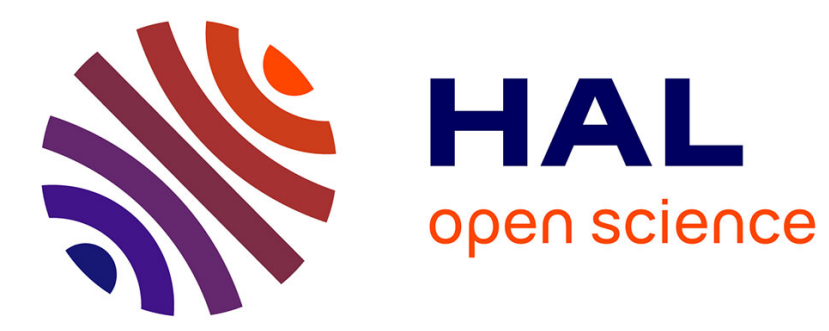

\title{
On Norms of Force Functionals and Stress Representations
}

\author{
Reuven Segev, Gal de Botton
}

\section{To cite this version:}

Reuven Segev, Gal de Botton. On Norms of Force Functionals and Stress Representations. Mathematics and Mechanics of Solids, 2006, 11 (3), pp.229-250. hal-01064978

\section{HAL Id: hal-01064978 \\ https://hal.science/hal-01064978}

Submitted on 17 Sep 2014

HAL is a multi-disciplinary open access archive for the deposit and dissemination of scientific research documents, whether they are published or not. The documents may come from teaching and research institutions in France or abroad, or from public or private research centers.
L'archive ouverte pluridisciplinaire HAL, est destinée au dépôt et à la diffusion de documents scientifiques de niveau recherche, publiés ou non, émanant des établissements d'enseignement et de recherche français ou étrangers, des laboratoires publics ou privés. 


\title{
ON NORMS OF FORCE FUNCTIONALS AND STRESS REPRESENTATIONS
}

\author{
R. SEGEV AND G. DEBOTTON JULY 14, 2003
}

\begin{abstract}
Forces in continuum mechanics are analyzed as 0-currents of geometric measure theory. The representation of forces by stresses is discussed and the flat norm of a force is expressed in terms of stress fields. An analogous treatment expresses the Sobolev norm of a force in terms of stress fields. In both cases, one obtain bounds on the stress fields that are in equilibrium with a given force. The analysis is universal in the sense that it is independent of any constitutive relation.
\end{abstract}

Keywords. Continuum mechanics, forces, stresses, norms, flat chains, divergence measures, Sobolev norms.

\section{INTRODUCTION}

This work presents some results of analysis in the context of force and stress theory of continuum mechanics. In particular, we consider various norms on the vector space of forces acting on a continuous body. The forces are regarded as continuous linear functionals defined on the vector space of virtual velocities. The norms of the forces we consider are the dual norms of the respective norms on the space of velocities.

The usual representation of the force functional in the form (we will use Einstein's summation convention in the sequel)

$$
F(w)=\int_{B} b_{i} w_{i} \mathrm{~d} v+\int_{\partial B} t_{i} w_{i} \mathrm{~d} a,
$$

where $w_{i}$ are the components of the virtual velocity field, suggests that the norm $\|w\|$ of the velocity field $w$ should depend only on the values of field $w$ in the body.

Date: July 14, 2003

Department of Mechanical Engineering, Ben-Gurion University, Beer Sheva 84105, Israel,

E-mail: rsegev@bgumail.bgu.ac.il, Fax: 972-7-6472813, Phone: 972-7-6477108. 
Thus for example, we may use the $C^{0}$-norm

$$
\|w\|_{C^{0}}=\sup _{i, x \in B}\left\{\left|w_{i}\right|\right\}
$$

for the velocity fields. This induces the dual norm for forces as

$$
\|F\|_{C^{0}}=\sup _{\|w\|_{C^{0}}=1}\{|F(w)|\}
$$

so the norm is the supremum of the power done by the force when it is evaluated on all velocity fields satisfying $\left|w_{i}(x)\right| \leqslant 1$. Since we may choose the velocity vector fields arbitrarily one obtains

$$
\|F\|_{C^{0}}=\sum_{i}\left(\int_{B}\left|b_{i}\right| \mathrm{d} v+\int_{\partial B}\left|t_{i}\right| \mathrm{d} a\right) .
$$

This way of measuring the magnitude of the force as a power functional is not satisfactory because we expect from experience that the virtual power should depend not only on the values of the velocity field but also on the values of its gradient $w_{i, m}$. This motivates using the $C^{1}$-norm on the space of velocities such that

$$
\|w\|_{C^{1}}=\sup _{x, i, m}\left\{\left|w_{i}(x)\right|,\left|w_{i, m}(x)\right|\right\}
$$

so the norm of the force,

$$
\|F\|_{C^{1}}=\sup _{\|w\|^{1}=1}\{|F(w)|\},
$$

is the supremum of the power done by the force when it is evaluated on all velocity fields satisfying both $\left|w_{i}(x)\right| \leqslant 1$ and $\left|w_{i, m}(x)\right| \leqslant 1$ for all $x \in B, i, m=1,2,3$.

The new expression for $\|F\|_{C^{1}}$ is not so straightforward to evaluate. In order to write an expression for it, we follow the method used by Federer [8, pp. 367-368] to represent Whitney's [15] flat norm of chains and we utilize the interpretation of the stress as a tensor valued measure representing a $C^{1}$-functional as in [13, 14]. Actually, in Section 3 we treat forces as vector valued flat 0 -chains on the body whose flat norm is expressed in terms of stresses. This may be described roughly as using the principle of virtual work

$$
F(w)=\int_{B} b_{i} w_{i} \mathrm{~d} v+\int_{\partial B} t_{i} w_{i} \mathrm{~d} a=\int_{B} \sigma_{i m} w_{i, m} \mathrm{~d} V,
$$

where, $\sigma_{i m}$ are the components of the stress tensor, to estimate the power expended by the force while the velocity gradient is bounded. While we know the power 
performed by the stresses only for compatible velocity gradient fields, the HahnBanach theorem is used estimate the work done by the stresses for non-compatible fields.

The method is presented in Section 2 and Section 3 considers in some detail the $C^{0}$ and $C^{1}$ norms described above. Section 4 considers the example of the Sobolev norms. Finally, the remarks of Section 5 suggest a situation where the foregoing analysis may be used to evaluate the results of structural optimization.

\section{REPRESENTATIONS OF DUAL NORMS}

The following is a straightforward generalization of Federer's [8, pp. 367-368] method of presentation of Whitney's [15] flat norm. It is analogous to the standard construction of the representation of elements in the dual spaces of the Sobolev spaces and distributions of finite order (e.g., $[11,16])$.

2.1. Basic settings. For a generic vector space $\mathbf{W}$ equipped with a seminorm $\|\cdot\|$ we consider a bounded linear functional $F: \mathbf{W} \rightarrow \mathbb{R}$, i.e., there is a positive number $C$ such that $|F(w)| \leqslant C\|w\|$ for all $w \in \mathbf{W}$. The dual norm of $F$ is defined by

$$
\|F\|=\sup _{\|w\|=1}\{|F(w)|\}=\sup _{w} \frac{|F(w)|}{\|w\|} .
$$

The representation of dual norms we consider in the next proposition applies to the following situation. Let $\mathbf{W}$ be a vector space with a seminorm $\|\cdot\|^{0}$ and $j: \mathbf{W} \rightarrow$ $J$, an injection into the vector space $J$ equipped with a seminorm $\|\cdot\|^{J}$. We consider the seminorm $\|\cdot\|$ on $\mathbf{W}$ defined by

$$
\|w\|=\|j(w)\|^{J}
$$

It is further assumed that $\|w\| \geqslant\|w\|^{0}$. The dual mapping $j^{*}: J^{*} \rightarrow \mathbf{W}^{*}$ satisfies $j^{*}(S)(w)=S(j(w))$, where we use $\mathbf{W}^{*}$ and $J^{*}$ to denote the spaces of linear functionals bounded relative to the seminorms on the respective spaces.

Proposition 2.1. For the situation described above, the dual norm of a linear functional $F$ on $\mathbf{W}$ bounded by $\|\cdot\|$ may be represented as

$$
\|F\|=\inf \left\{\left\|F-j^{*}(S)\right\|^{0}+\|S\|^{J}\right\},
$$


where the infimum it taken over all linear functionals $S$ on $J$ bounded by $\|\cdot\|^{J}$. There is at least one $\hat{S} \in J^{*}$ satisfying $F=j^{*}(\hat{S})$ for which the infimum in the expression for $\|F\|$ is attained, so

$$
\|F\|=\inf _{F=j^{*}(S)}\left\{\|S\|^{J}\right\} .
$$

Proof. We first note that by the assumptions above for any linear functional $S: J \rightarrow$ $\mathbb{R}$ bounded by $\|\cdot\|^{J}$ we have

$$
\left|j^{*}(S)(w)\right|=|S(j(w))| \leqslant\|S\|^{J}\|j(w)\|^{J}=\|S\|^{J}\|w\|,
$$

so $j^{*}(S)$ on $\mathbf{W}$ is bounded. In addition,

$$
\begin{aligned}
\left\|j^{*}(S)\right\| & =\sup _{w} \frac{\left|j^{*}(S)(w)\right|}{\|w\|} \\
& =\sup _{w} \frac{|S(j(w))|}{\|w\|} \\
& \leqslant \sup _{w} \frac{\|S\|^{J}\|j(w)\|^{J}}{\|j(w)\|^{J}},
\end{aligned}
$$

so $\left\|j^{*}(S)\right\| \leqslant\|S\|^{J}$. Now, given a functional $F$, for every $S \in J^{*}$

$$
F(w)=\left(F-j^{*}(S)\right)(w)+S(j(w))
$$

thus,

$$
\begin{aligned}
\|F\| & =\sup _{\|w\|=1}\{|F(w)|\} \\
& \leqslant \sup _{\|w\|=1}\left\{\left|\left(F-j^{*}(S)\right)(w)\right|\right\}+\sup _{\|w\|=1}\{|S(j(w))|\} \\
& \leqslant \sup _{\|w\|^{0}=1}\left\{\left|\left(F-j^{*}(S)\right)(w)\right|\right\}+\sup _{\|w\|=1}\{|S(j(w))|\} \\
& \leqslant\left\|F-j^{*}(S)\right\|^{0}+\|S\|^{J},
\end{aligned}
$$

where we used the fact that $\|w\|^{0} \leqslant\|w\|$ to arrive at the third line and we used the fact that $\|w\|=1$ implies $\|j(w)\|^{J}=1$ to arrive at the fourth line. It should be noted that $\left\|F-j^{*}(S)\right\|^{0}$ might be infinite and in general $\|F\|^{0} \geqslant\|F\|$.

We now show that the inequality is exact. Note that for every $\psi \in \operatorname{Image} j$ we have $\left|F\left(j^{-1}(\psi)\right)\right| \leqslant\|F\|\left\|j^{-1}(\psi)\right\|$ so

$$
F \circ j^{-1}: \text { Image } j \subset J \longrightarrow \mathbb{R}
$$


is a linear functional that is bounded relative to the seminorm $\|\cdot\|^{J}$. By the HahnBanach theorem, $F \circ j^{-1}$ may be extended to a linear functional $\hat{S}: J \rightarrow \mathbb{R}$ such that $F(w)=\hat{S}(j(w))$. Hence, $F=j^{*}(\hat{S})$ and $|\hat{S}(\psi)| \leqslant\|F\|\|\psi\|^{J}$ for all $\psi \in J$ so $\|\hat{S}\|^{J} \leqslant\|F\|$. In general, if $F=j^{*}(S)$, then $\|S\|^{J} \geqslant\|F\|$. We conclude that $\|\hat{S}\|^{J}=\|F\|$.

Since $j^{*}$ is surjective, we note that the representation theorem may be written alternatively as

$$
\|F\|=\inf \left\{\|G\|^{0}+\|S\|^{J}\right\}
$$

where the infimum is taken all representations of $F$ in the form

$$
F=G+j^{*}(S)
$$

where $G: \mathbf{W} \rightarrow \mathbb{R}$ and $S: J \rightarrow \mathbb{R}$ are linear and bounded by the $\|\cdot\|^{0}$ and $\|\cdot\|^{J}$ norms, respectively.

Using the notation above, we now add some additional structure.

Proposition 2.2. Assume that $J=J_{0} \times J_{1}$ where the spaces $J_{0}$ and $J_{1}$ are equipped with seminorms $\|\cdot\|^{J_{0}}$ and $\|\cdot\|^{J_{1}}$, respectively, and for $\psi=\left(\psi_{0}, \psi_{1}\right) \in J$

$$
\left(\|\psi\|^{J}\right)^{p}=\left(\left\|\psi_{0}\right\|^{J_{0}}\right)^{p}+\left(\left\|\psi_{1}\right\|^{J_{1}}\right)^{p}, \quad 1 \leqslant p \leqslant \infty,
$$

where for $p=\infty$ it is understood that $\|\psi\|^{J}=\max \left\{\left\|\psi_{0}\right\|^{J_{0}},\left\|\psi_{1}\right\|^{J_{1}}\right\}$.

Then,

$$
\|F\|=\inf \left\{\left(\left\|F-j_{1}^{*}\left(S_{1}\right)\right\|^{J_{0}}\right)^{q}+\left(\left\|S_{1}\right\|^{J_{1}}\right)^{q}\right\}^{1 / q}, \quad \frac{1}{p}+\frac{1}{q}=1,
$$

where the infimum is taken over all linear functionals $S_{1}$ on $J_{1}$ bounded relative to $\|\cdot\|^{J_{1}}$.

Proof. As in the previous proposition $F$ may be represented by some $\hat{S} \in J^{*}$ such that $F=j^{*}(S)$, with $\|F\|=\|S\|^{J}$. The product structure implies that $\hat{S}$ is of the form $\hat{S}=\left(\hat{S}_{0}, \hat{S}_{1}\right), \hat{S}_{\gamma} \in J_{\gamma}^{*}, \gamma=0,1,\left(\|\hat{S}\|^{J}\right)^{q}=\left(\left\|\hat{S}_{0}\right\|\right)^{q}+\left(\left\|\hat{S}_{1}\right\|\right)^{q}$, so

$$
F(w)=\hat{S}_{0}\left(j_{0}(w)\right)+\hat{S}_{1}\left(j_{1}(w)\right), \quad F=j_{0}^{*}\left(\hat{S}_{0}^{*}\right)+j_{1}^{*}\left(\hat{S}_{1}^{*}\right) .
$$


Since any $S$ such that $F=j^{*}(S)$ may be written in the form $S=\left(F-j_{1}^{*}\left(S_{1}\right), S_{1}\right)$,

$$
\begin{aligned}
(\|F\|)^{q} & =\inf _{F=j^{*}(S)}\left\{\left(\|S\|^{J}\right)^{q}\right\} \\
& =\inf _{S_{1} \in J_{1}^{*}}\left\{\left(\left\|\left(F-j_{1}^{*}\left(S_{1}\right), S_{1}\right)\right\|^{J}\right)^{q}\right\} \\
& =\inf _{S_{1} \in J_{1}^{*}}\left\{\left(\left\|F-j_{1}^{*}\left(S_{1}\right)\right\|^{J_{0}}\right)^{q}+\left(\left\|S_{1}\right\|^{J_{1}}\right)^{q}\right\} .
\end{aligned}
$$

Remark 2.3. In the following we will be concerned with the mapping $j: w \mapsto$ $(w, \nabla w)$, where $\nabla w$ denotes the gradient of $w$. Thus, $j_{1}=\nabla$-the gradient operator, and $j_{1}^{*}=\partial$, the boundary operator defined by $\partial S(w)=S(\nabla w)$. Hence, may write for this case

$$
\left.(\|F\|)^{q}=\inf _{S_{1} \in J_{1}^{*}}\left\{\left(\| F-\partial S_{1}\right) \|^{J_{0}}\right)^{q}+\left(\left\|S_{1}\right\|^{J_{1}}\right)^{q}\right\} .
$$

\section{The Flat NORMS OF FORCE FunCtiOnALS}

3.1. The force functional. Consider a body $B$ which is assumed to be a smooth compact 3-dimensional submanifold with boundary of $\mathbb{R}^{3}$. For simplicity we also identify the physical space with $\mathbb{R}^{3}$ and identify the body with its current configuration in $\mathbb{R}^{3}$. In traditional continuum mechanics it is assumed that a force $F$ on $B$ is given in terms of a body force field $b: B \rightarrow \mathbb{R}^{3}$ and a surface force field $t: \partial B \rightarrow \mathbb{R}^{3}$. Assume that these fields are integrable functions relative to the volume and area measures respectively. The force $F$ induces a linear functional-the virtual power functional—on the space of continuous vector fields $C^{0}\left(B, \mathbb{R}^{3}\right)$. We use the same notation $F$ for the induced functional and it is defined by

$$
F(w)=\int_{B} b_{i} w_{i} \mathrm{~d} V+\int_{\partial B} t_{i} w_{i} \mathrm{~d} A .
$$

The vector fields $w$ are interpreted physically as virtual displacement or virtual velocity fields and $F(w)$ is interpreted as the virtual power.

We may regard the body as a measure space $B=\operatorname{Int} B \cup \partial B$ equipped with the Radon measure $\mu$ such that

$$
\mu(P)=V(P \cap \operatorname{Int} B)+A(P \cap \partial B),
$$


where $V$ is the volume measure in $\operatorname{Int} B$ and $A$ is the area measure on $\partial B$. Thus,

$$
\int_{B} u \mathrm{~d} \mu=\int_{\text {Int } B} u \mathrm{~d} V+\int_{\partial B} u \mathrm{~d} A
$$

for any integrable function $u$. Since $\partial B$ is of volume measure zero, we will often simplify the notation and omit the indication of the interior for the domain of the volume integral. We may regard the force fields as the restrictions to Int $B$ and $\partial B$ of a vector field $f$ on $B$ such that $f(x)=b(x)$ for $x \in \operatorname{Int} B$ and $f(x)=t(x)$ for $x \in \partial B$, so

$$
F(w)=\int_{B} f_{i} w_{i} \mathrm{~d} \mu
$$

where $f$ is integrable relative to $\mu$. Thus, we may regard the force $F$ as a measure on $B$ which is absolutely continuous relative to the measure $\mu$ whose density is the vector field $f$, i.e., $F=f \cdot \mu$-the product of the measure $\mu$ by the integrable function $f$.

3.2. The $C^{0}$-norm of a force functional. The force functional $F$ of continuum mechanics is continuous relative to the norm

$$
\|w\|_{C^{0}}=\sup \left\{\left|w_{i}(x)\right| ; x \in B, i=1,2,3\right\}
$$

on $C^{0}\left(B, \mathbb{R}^{3}\right)$. As a member of the normed dual, for a force given in terms of a body force and a surface force,

$$
\|F\|_{C^{0}}=\sup _{\|w\|_{C^{0}}=1}\left|\int_{B} b_{i} w_{i} \mathrm{~d} V+\int_{\partial B} t_{i} w_{i} \mathrm{~d} a\right| .
$$

Remark 3.1. In terms of geometric measure theory (c.f. $[8,9]$ ) a force is a (vector valued) 0 -current that is represented by integration. The norm $\|\cdot\|_{C^{0}}$ is the mass norm of the force.

Since $F$ is represented by the measure $f \cdot \mu$, the Riesz representation theorem and the independence of the components $w_{i}$ imply that $\|F\|_{C^{0}}=\sum_{i}\left|f_{i} \cdot \mu\right|(B)$, where $\left|f_{i} \cdot \mu\right|$ denotes the total variation of the respective measure. Since $\mu$ is positive, $\left|f_{i} \cdot \mu\right|=\left|f_{i}\right| \cdot \mu$ so

$$
\left|f_{i} \cdot \mu\right|(B)=\int_{B}\left|f_{i}\right| \mathrm{d} \mu=\int_{B}\left|b_{i}\right| \mathrm{d} V+\int_{\partial B}\left|t_{i}\right| \mathrm{d} A
$$

and 


$$
\|F\|_{C^{0}}=\sum_{i} \int_{B}\left|b_{i}\right| \mathrm{d} V+\sum_{i} \int_{\partial B}\left|t_{i}\right| \mathrm{d} A .
$$

3.3. The $C^{1}$-norm of virtual velocities. The principle of virtual work in continuum mechanics represents the equations of equilibrium for the components $\sigma_{i m}$ of the stress tensor in the weak form

$$
F(w)=\int_{B} b_{i} w_{i} \mathrm{~d} V+\int_{\partial B} t_{i} w_{i} \mathrm{~d} V=\int_{B} \sigma_{i m} w_{i, m} \mathrm{~d} V,
$$

for all differentiable virtual velocity vector fields $w$. As mentioned in the introduction, this expression implies that the magnitude of a force functional should be defined in such a way so the magnitude of the derivatives of the virtual velocity fields is reflected. In other words, while the traditional representation of a force in terms of a body force field and a surface force field suggests, as we did in the preceding subsection, that its magnitude should be evaluated using the $C^{0}$-norm, the principle of virtual work suggests using the $C^{1}$-norm

$$
\|w\|_{C^{1}}=\sup _{x, i, m}\left\{\left|w_{i}(x)\right|,\left|w_{i, m}\right|\right\} .
$$

In fact, the mere requirement that a force be an element of $C^{1}\left(B, \mathbb{R}^{3}\right)^{*}$ implies through a representation theorem for such functionals several features we expect of stress theory of continuum mechanics (see $[13,14])$ some of which we will see below.

Our objective in the rest of this section is to regard a force in continuum mechanics as an element of $C^{1}\left(B, \mathbb{R}^{3}\right)^{*}$ that in addition is a member of $C^{0}\left(B, \mathbb{R}^{3}\right)^{*}$. In particular, we will consider the representation of such forces in terms of a body force and a surface force fields.

The representation of dual norms we consider in the rest of this section applies to the following situation. Let $\mathbf{W}$ be a vector space with a norm $\|\cdot\|^{0}$ and $j: \mathbf{W} \rightarrow$ $J=\prod_{\alpha} J^{\alpha}, \alpha \in a$ an injection. Here, $J^{\alpha}$ are vector spaces with seminorms $\|\cdot\|^{(\alpha)}$ so we have the seminorm $\|\cdot\|^{J}$ given by $\|\psi\|^{J}=\sup _{\alpha}\left\|\psi_{\alpha}\right\|$. Thus, the seminorm $\|\cdot\|$ on $\mathbf{W}$ is given by

$$
\|w\|=\|j(w)\|^{J}=\sup _{\alpha}\left\{\left\|j^{\alpha}(w)\right\|^{(\alpha)}\right\}
$$


where $j^{\alpha}$ is the $\alpha$-component of $j$. It is further assumed that $\|w\| \geqslant\|w\|^{0}$ so $\|\cdot\|$ is also a norm (for example, when only a subset of $a$ is used to define $\|\cdot\|^{0}$ ). The dual mapping $j^{*}: J^{*} \rightarrow \mathbf{W}^{*}$ satisfies $j^{*}(S)(w)=S(j(w))=\sum_{\alpha} S_{\alpha}\left(j^{\alpha}(w)\right)=$ $\sum_{\alpha} j^{*}\left(S_{\alpha}\right)(w)$, where $S_{\alpha}, \alpha \in a$, are the components of $S \in J^{*}$.

Thus, we use the notation and results of Section 2 for the case where $\mathbf{W}=$ $C^{1}\left(B, \mathbb{R}^{3}\right)$ and the initial norm $\|\cdot\|^{0}$ is the $C^{0}$-norm as above. The space $J$ is given by

$$
J=\prod_{\alpha \in a} J^{\alpha}, \quad J^{\alpha}=C^{0}(B, \mathbb{R})
$$

and the index set is

$$
a=\{\alpha=(i, k), i \in\{1,2,3\}, k \in\{0,1,2,3\}\} .
$$

(In the sequel, the index $k$ will range from 0 to 3.) On $J^{\alpha}$ we use the norm $\left\|\psi^{\alpha}\right\|^{(\alpha)}=$ $\sup _{x \in B}\left|\psi^{\alpha}(x)\right|$. Thus, we make the identification $J=C^{0}\left(B, \mathbb{R}^{12}\right)$.

The mapping $j: C^{1}\left(B, \mathbb{R}^{3}\right) \rightarrow J$ is defined by its components $j^{\alpha}$ as follows. For $\alpha=(i, k)$, we set

$$
j^{\alpha}: C^{1}\left(B, \mathbb{R}^{3}\right) \rightarrow C^{0}(B, \mathbb{R})
$$

as

$$
j^{i 0}(w)=w_{i}=w_{i, 0}, \quad \text { and for } k \neq 0 \quad j^{i k}(w)=w_{i, k} .
$$

Thus, for $w \in C^{1}\left(B, \mathbb{R}^{3}\right)$,

$$
\|w\|=\sup _{x, i, k}\left\{\left|w_{i, k}(x)\right|\right\}=\|w\|_{C^{1}} .
$$

Clearly, $\|w\|^{0} \leqslant\|w\|$ for all fields $w$.

3.4. Representation of forces by stresses. As stated above, we consider forces defined as linear functionals on velocity fields that are bounded by both the $C^{0}$ and the $C^{1}$ norms of the velocity fields. In terms of geometric measure theory, the requirement that a force be bounded by both norms makes it a flat chain. Its $C^{1}$-norm is its flat norm.

According to the setting described above and using Proposition 2.1, for each force $F$ there is an element $S \in J^{*}$ such that $F(w)=S(j(w))$. Since $J$ is a product space, $S$ is represented by its components $S_{\alpha}$ as $S(\psi)=\sum_{\alpha} S_{\alpha}\left(\psi_{\alpha}\right)$. Thus, $F(w)=\sum_{\alpha} S_{\alpha}\left(j^{\alpha}(w)\right)$, and $F=\sum_{\alpha} j^{\alpha *}\left(S_{\alpha}\right)$. We refer to the element $S \in J^{*}$ as a stress representing the force $F$. 
The Riesz representation theorem implies that every linear functional $S_{\alpha}$ on $J^{\alpha}=C^{0}(B, \mathbb{R})$ that is continuous relative to the $C^{0}$-norm may be represented as an integral

$$
S_{\alpha}\left(\psi_{\alpha}\right)=\int_{B} \psi_{\alpha} \mathrm{d} s_{\alpha}, \quad(\text { no sum on } \alpha) .
$$

relative to a Radon measure $s_{\alpha}$ and $\left\|S_{\alpha}\right\|_{C^{0}}=\left|s_{\alpha}\right|(B)$. It follows that for any given $S$ there is a collection $\left\{s_{\alpha}\right\}$ of measures such that

$$
S(\psi)=\int_{B} \psi_{\alpha} \mathrm{d} s_{\alpha}
$$

and $\|S\|^{J}=\|S\|_{C^{0}}=\sum_{\alpha}\left|s_{\alpha}\right|(B)$. Thus,

$$
\begin{aligned}
j^{*}(S)(w) & =\int_{B} w_{i, k} d s_{i k}, & k=0,1,2,3, \\
& =\int_{B} w_{i} \mathrm{~d} s_{i 0}+\int_{B} w_{i, m} \mathrm{~d} s_{i m}, & m=1,2,3 .
\end{aligned}
$$

(Henceforth, we will use $k$ for an index ranging from 0 to 3 and $m$ for an index ranging from 1 to 3.) Proposition 2.1 implies that given $F \in C^{0}\left(B, \mathbb{R}^{3}\right) \cap C^{1}\left(B, \mathbb{R}^{3}\right)$ there is a collection of stress measures $s_{\alpha}$ such that

$$
F(w)=\int_{B} w_{i} \mathrm{~d} s_{i 0}+\int_{B} w_{i, m} \mathrm{~d} s_{i m}=j^{*}(S)(w),
$$

which is a generalized form of the principle of virtual work. We note that the terms involving $s_{i 0}$ which do not appear in the traditional formulation of stress theory, do appear in generalized formulations of continuum mechanics and are sometimes referred to as "self forces" (e.g., see Capriz [5]).

The assumption that $F$ is representable by integration, i.e., $\|F\|_{C^{0}}$ is finite, implies that the stress components $S_{1 i}=\left\{S_{i 1}, S_{i 2}, S_{i 3}\right\}$, satisfy the condition

$$
\sup _{\left\|w_{i}\right\|^{0}=1}\left\{\left|S_{i m}\left(w_{i, m}\right)\right|\right\}=\sup _{\left|w_{i}\right| \leqslant 1}\left\{\left|\int_{B} w_{i, m} \mathrm{~d} s_{i m}\right|\right\}<\infty, \quad \text { no sum on } i .
$$

In other words, the boundary $\partial S_{1 i}, \partial S_{1 i}\left(w_{i}\right)=S_{i m}\left(w_{i, m}\right)$ (no sum on $i$ ), is also representable by integration. In the terminology of geometric measure theory, as both $S_{1 i}$ and its boundary $\partial S_{1 i}$ are representable by integration, $S_{1 i}$ is a normal 1-current. 
Let $s$ be a measure on $B$. Then, we may decompose $s$ into the two measures $\left.s\right|_{\partial B}$ on the boundary of $B$ and a measure $\left.s\right|_{\operatorname{Int} B}$ on the interior of $B$. We have $s(D)=$ $\left.s\right|_{\partial B}(D \cap \partial B)+\left.s\right|_{\operatorname{Int} B}(D \cap \operatorname{Int} B)$ for every measurable subset $D$. In addition, for every s-integrable function $\phi$

$$
\int_{B} \phi \mathrm{d} s=\left.\int_{\partial B} \phi \mathrm{d} s\right|_{\partial B}+\left.\int_{\operatorname{Int} B} \phi \mathrm{d} s\right|_{\operatorname{Int} B} .
$$

By the additivity of the total variation measure

$$
|s|_{\partial B}|=| s||_{\partial B^{\prime}}|s|_{\operatorname{Int} B}|=| s||_{\operatorname{Int} B} .
$$

In particular, $\|s\|_{C^{0}}=|s|(B)=\left|s\left\|_{\operatorname{Int} B}(\operatorname{Int} B)+\mid s\right\|_{\partial B}(\partial B)\right.$. If $\sigma$ and $\tau$ are smooth densities on $\operatorname{Int} B$ and $\partial B$ representing $\left.s\right|_{\operatorname{Int} B}$ and $\left.s\right|_{\partial B}$ relative to the volume and area measures, respectively, then

$$
\|S\|_{C^{0}}=\int_{\text {Int } B}|\sigma| \mathrm{d} V+\int_{\partial B}|\tau| \mathrm{d} A .
$$

Thus, considering the stress measures, for any fixed $i$,

$$
\partial S_{1 i}\left(w_{i}\right)=\left.\int_{\partial B} w_{i, m} \mathrm{~d} s_{i m}\right|_{\partial B}+\left.\int_{\operatorname{Int} B} w_{i, m} \mathrm{~d} s_{i m}\right|_{\operatorname{Int} B} .
$$

Restricting $\partial S_{1 i}$ to functions $\phi$ compactly supported in Int $B$ it is clear that

$$
\sup _{\|\phi\|_{\mathcal{C}^{0}}=1}\left\{\left|s_{1 i m}\right|_{\operatorname{Int} B}(\phi, m) \mid\right\}<\infty, \quad \operatorname{Supp} \phi \subset \operatorname{Int} B,
$$

which we will use in the next subsection.

3.5. The Green-Gauss Theorem of Divergence Measures. We now use the results of a recent work [7] by Chen and Frid. Consider a finite $\mathbb{R}^{3}$-valued Radon measure $\mu=\left\{\mu_{i}\right\}$ defined on an open set $U \subset \mathbb{R}^{3}$. The measure $\mu$ is regarded as a (vector valued) distribution on $U$ so its distributional divergence $\operatorname{div} \mu$ is defined by

$$
\operatorname{div} \mu(\phi)=-\int_{U} \phi_{, i} \mathrm{~d} \mu_{i}
$$

for all differentiable functions $\phi$ compactly supported in $U$. In the terminology introduced by [7], the finite measure $\mu$ is defined to be an extended divergence measure if

$$
|\operatorname{div} \mu|(U):=\sup _{\|\psi\|_{\mathbb{C}^{0}}=1}\{\operatorname{div} \mu(\psi)\}<\infty
$$


for all differentiable functions $\psi$ compactly supported in $U$, so that $\operatorname{div} \mu$ is a finite measure on $U$. Thus, in the language of geometric measure theory, $\mu$ represents a locally normal current. Being a finite measure on $U, \operatorname{div} \mu$ may be extended to a Radon measure on $\mathbb{R}^{3}$.

The generalized Green-Gauss theorem is concerned with the difference between - $\operatorname{div} \mu$, where $\operatorname{div} \mu$ is considered as a distribution on $\mathbb{R}^{3}$, and $\partial \mu$, where we adopt the notation that when we write $\partial \mu$ we regard $\mu$ as a current on $\mathbb{R}^{3}$ (cf. $[9$, pp. 138139]). Thus, for differentiable functions $\psi$ compactly supported in $\mathbb{R}^{3}$, one is looking for an expression for $\partial \mu(\psi)+\operatorname{div} \mu(\psi)$.

Restricting the discussion of [7] to the case considered here where the open set is Int $B$ and $\partial B$ is smooth, the generalized Green-Gauss theorem presents the difference between $\partial \mu$ and $-\operatorname{div} \mu$ as a continuous linear functional $\mu_{i} v_{i}$ defined on $C^{1}\left(\partial B, \mathbb{R}^{3}\right)$, called the normal trace of $\mu$ on the boundary. At this stage, the notation $\mu_{i} v_{i}$ is formal and the expression for the normal trace is given below. Here, $v$ denotes the outwards pointing unit normal to $\partial B$. Thus, one may write,

$$
\partial \mu(\psi)+\operatorname{div} \mu(\psi)=\mu_{i} v_{i}\left(\left.\psi\right|_{\partial B}\right) .
$$

where $\psi$ is any differentiable function on $\mathbb{R}^{3}$.

3.6. The Normal Trace for Smooth Boundaries. The assumption that $\partial B$ is smooth allows additional simplification of the results of [7] as follows. The mapping $\Psi:(y, n) \mapsto y+n v$ is a diffeomorphism of $\partial B \times[-\varepsilon, \varepsilon]$ for some $\varepsilon>0$ onto some neighborhood of $\partial B$ in $\mathbb{R}^{3}$ (e.g., a normal tubular neighborhood as in [10, p. 110]). The mapping $h=\Psi_{2}^{-1}: x \mapsto n(x)$, the second component of $\Psi^{-1}$, may serve with minor modifications as the function denoted by $h$ in [7, Theorem 3.1]. Clearly, $\nabla h=v$, i.e., $v$ is the gradient of the distance function.

Let $H$ be a smooth function on $\mathbb{R}$ such that $H$ is supported in $[-\varepsilon, \varepsilon], H(0)=1$, and $0 \leqslant H(x) \leqslant 1$. In particular, one can choose $H$ such that $H(n)=1$ for all $n$ in a neighborhood of 0 in $[-\varepsilon, \varepsilon]$ so the derivative of $H$ vanishes at 0 . A simple extension $\mathcal{E}(\psi) \in C^{1}\left(\mathbb{R}^{3}, \mathbb{R}\right)$ of $\psi \in C^{1}(\partial B, \mathbb{R})$ to $\mathbb{R}^{3}$ may be defined by $\mathcal{E}(\psi)(x)=\psi(y) H(n)$ for $x \in \operatorname{Image} \Psi$, so $|n| \leqslant \varepsilon$, and $\mathcal{E}(\psi)(x)=0$ elsewhere. With the choice of $H$ that is constant in a neighborhood of $n=0, \mathcal{E}(\psi)(\Psi(y, n))=$ $\psi(y)$ in that neighborhood. Clearly, once one chooses $H$ with these properties, 
$\|\mathcal{E}(\psi)\|_{C^{0}}=\|\psi\|_{C^{0}}$. Writing

$$
\begin{aligned}
\nabla(\mathcal{E}(\psi))(y, n) & =\left(\nabla_{\partial} \mathcal{E}(\psi), \mathcal{E}(\psi), v\right)(y, n) \\
& =\left(\nabla_{\partial} \psi(y) H(n), \psi(y) H^{\prime}(n)\right),
\end{aligned}
$$

where $\nabla_{\partial} \psi$ is the 2-dimensional gradient of $\psi$, shows that $\|\mathcal{E}(\psi)\|_{C^{1}} \leqslant K\|\psi\|_{C^{1}}$ for some constant $K$. (It is noted that in extending a function $\psi$ defined on a closed set to $\mathbb{R}^{3}$ according to Whitney's theorem, all the derivatives $\psi_{, i}, i=1,2,3$ on $\partial B$ should be specified. We arbitrarily choose the independent normal derivative to vanish.)

In our notation, the expression for the normal trace in [7] is

$$
\left.\mu_{i} v_{i}\right|_{\partial B}(\psi)=\lim _{n_{0} \rightarrow 0^{-}} \frac{1}{n_{0}} \int_{\Psi\left(\partial B \times\left(n_{0}, 0\right)\right)} \mathcal{E}(\psi) v_{i} \mathrm{~d} \mu_{i}
$$

for any differentiable $\psi$ defined on $\partial B$. Using the particular choice of $H$ as above this simplifies to

$$
\left.\mu_{i} v_{i}\right|_{\partial B}(\psi)=\lim _{n_{0} \rightarrow 0^{-}} \frac{1}{n_{0}} \int_{\Psi\left(\partial B \times\left(n_{0}, 0\right)\right)} \psi(y) v_{i}(y) \mathrm{d} \mu_{i}(\Psi(y, n)) .
$$

It follows that $\left.\mu_{i} v_{i}\right|_{\partial B}(\psi)$ is bounded by $\sup _{y \in \partial B}\{|\psi(y)|\}$. Hence, for the case of a smooth boundary, $\mu_{i} v_{i}$ is a Radon measure on $\partial B$, i.e., $\mu_{i} v_{i} \in C^{0}(\partial B, \mathbb{R})^{*} \subset$ $C^{1}(\partial B, \mathbb{R})^{*}$ and we may write $\mu_{i} v_{i}\left(\left.\phi\right|_{\partial B}\right)$ as $\int_{\partial B} \phi \mathrm{d}\left(\mu_{i} v_{i}\right)$.

We now apply the generalized Green-Gauss theorem for extended divergence measures to $\left.s_{i m}\right|_{\operatorname{Int} B}$ and we incorporate the observations made above. Since we consider forces in $C^{1}\left(B, \mathbb{R}^{3}\right)^{*} \cap C^{0}\left(B, \mathbb{R}^{3}\right)^{*}$, restricting the expression for the representation of forces by stress measures to fields supported in Int $B$ we conclude that for each $i$ the collection $\left\{s_{i m}, m=1,2,3\right\}$ is a divergence measure. Therefore,

$$
\left.\int_{\text {Int } B} w_{i, m} \mathrm{~d} s_{i m}\right|_{\operatorname{Int} B}=\int_{\partial B} w_{i} \mathrm{~d}\left(s_{i m} v_{m}\right)-\int_{\operatorname{Int} B} w_{i} \mathrm{~d} s_{i m, m},
$$

where we use $s_{i m, m}$ to denote $\left.\operatorname{div} S_{1 i}\right|_{\operatorname{Int} B}$.

3.7. Analysis of the Stress on $\partial B$. With the decomposition of the stress measure to measures on Int $B$ and measures on $\partial B$ and using the Generalized Green-Gauss 
theorem the representation of the force $F$ assumes the form

$$
\begin{aligned}
F(w)= & \left.\int_{\partial B} w_{i} \mathrm{~d} s_{i 0}\right|_{\partial B}+\left.\int_{\partial B} w_{i, m} \mathrm{~d} s_{i m}\right|_{\partial B}+\left.\int_{\operatorname{Int} B} w_{i} \mathrm{~d} s_{i 0}\right|_{\operatorname{Int} B} \\
& +\int_{\partial B} w_{i} \mathrm{~d}\left(s_{i m} v_{m}\right)-\int_{\operatorname{Int} B} w_{i} \mathrm{~d} s_{i m, m} .
\end{aligned}
$$

We first use the decomposition of vectors on $\partial B$ into tangential and normal components to represent $w_{i, m}$ by $\left(\nabla_{\partial} w_{i}, w_{i, v}\right)$, where $w_{i, v}$ is the derivative of $w_{i}$ in the direction of the unit normal. Accordingly, we may represent $\left.s_{i m}\right|_{\partial B}$ by the measures $\left(T_{i \partial}, T_{i v}\right)$ on $\partial B$ so that

$$
\left.\int_{\partial B} w_{i, m} \mathrm{~d} s_{i m}\right|_{\partial B}=\int_{\partial B} \nabla_{\partial} w_{i} \mathrm{~d} T_{i \partial}+\int_{\partial B} w_{i, v} \mathrm{~d} T_{i v}, \quad \text { no sum on } i \text {. }
$$

The expression for the force becomes

$$
\begin{aligned}
F(w)= & \left.\int_{\partial B} w_{i} \mathrm{~d} s_{i 0}\right|_{\partial B}+\int_{\partial B} \nabla_{\partial} w_{i} \mathrm{~d} T_{i \partial}+\int_{\partial B} w_{i, \nu} \mathrm{d} T_{i v}+\left.\int_{\operatorname{Int} B} w_{i} \mathrm{~d} s_{i 0}\right|_{\operatorname{Int} B} \\
& +\int_{\partial B} w_{i} \mathrm{~d}\left(s_{i m} v_{m}\right)-\int_{\operatorname{Int} B} w_{i} \mathrm{~d} s_{i m, m} .
\end{aligned}
$$

Let $\psi$ be a differentiable function on $\partial B$. Using a normal tubular neighborhood, for every $\delta>0$ one can construct a differentiable function $\phi$ on $B$ such that $\phi(y)=0, \phi, v(y)=\psi(y)$ for $y \in \partial B$, and $\sup _{x \in B}|\phi(x)|<\delta$. Applying the expression for the representation of $F$ to a vector field $w$ whose $i$-th component is $\phi$ and the other components vanish with taking the limit as $\delta \rightarrow 0$, only the terms containing the normal derivative remain finite $\left(\nabla_{\partial} w\right.$ vanishes on the boundary by the construction) so that

$$
\left|\int_{\partial B} \psi \mathrm{d} T_{i v}\right| \leqslant K \delta
$$

for some constant $K$. Since any continuous function on the boundary may be uniformly approximated by a differentiable function, it follows that $T_{i v}=0$.

Next, since in the resulting representation of $F$,

$$
\int_{\partial B} \nabla_{\partial} w_{i} \mathrm{~d} T_{i \partial}
$$

is the only term that contains the derivative, it is also bounded by $\sup _{x \in B}\{|w(x)|\}$. Let $\psi$ be a differentiable function on $\partial B$. Using the extension of Paragraph 3.6 we 
may extend it to a function $\phi$ on $B$ such that

$$
\sup _{x \in B}\{|\phi(x)|\}=\sup _{y \in \partial B}\{|\psi(y)|\}
$$

Hence,

$$
\int_{\partial B} \nabla_{\partial} \phi \mathrm{d} T_{i \partial}
$$

is bounded by $\sup _{y \in \partial B}\{|\psi(y)|\}$ and $T_{i \partial}$ is a normal current on $\partial B$. Since $\partial(\partial B)=\varnothing$ normal currents are divergence measures and we may write ${ }^{1}$

$$
\left.\int_{\partial B} \nabla_{\partial} w_{i} \mathrm{~d} T_{i \partial}=-\int_{\partial B} w_{i} \mathrm{~d}\left(\operatorname{div}_{\partial} T_{i \partial}\right), \quad \text { (no sum on } i\right) .
$$

We conclude that

$$
\left.\int_{\partial B} w_{i, m} \mathrm{~d} s_{i m}\right|_{\partial B}=-\int_{\partial B} w_{i} \mathrm{~d}\left(\operatorname{div} \partial T_{i \partial}\right)
$$

3.8. The Representation of Force Measures. We now combine the observations of the preceding paragraphs to obtain a representation of forces in $C^{0}\left(B, \mathbb{R}^{3}\right)^{*} \cap$ $C^{1}\left(B, \mathbb{R}^{3}\right)^{*}$ in terms of the stress measures as

$$
F(w)=j^{*}(S)(w)=\int_{\operatorname{Int} B} w_{i} \mathrm{~d}\left(s_{i 0}-s_{i m, m}\right)+\int_{\partial B} w_{i} \mathrm{~d}\left(s_{i 0}-\operatorname{div} \partial T_{i \partial}+s_{i m} v_{m}\right)
$$

It is noted that this representation applies to any element of $C^{0}\left(B, \mathbb{R}^{3}\right)^{*} \cap C^{1}\left(B, \mathbb{R}^{3}\right)^{*}$ and it need not be represented a-priori in terms of a body force field and a surface force field. In addition, the representation contains surface tension terms.

In case the force $F$ is given in terms of a body force field $b_{i}$ and a surface force field $t_{i}$ we obtain the following conditions for $F$ to be represented by the stress measures:

$$
b_{i} \cdot V=s_{i 0}-s_{i m, m}, \quad t_{i} \cdot A=s_{i 0}-\operatorname{div}_{\partial} T_{i \partial}+s_{i m} v_{m} .
$$

\footnotetext{
${ }^{1}$ We may use here the extension of the generalized Green-Gauss theorem to Riemannian manifolds (see [7], Remark 3.4).
} 
3.9. The $C^{1}$-Norm of Forces. In the terminology of geometric measure theory the $C^{1}$-norm of the force is its flat norm. In order to use the expression

$$
\|F\|=\sup \left\{\left\|F-j^{*}(S)\right\|^{0}+\|S\|^{J}\right\}
$$

for the flat norm of a force given in terms of a body force $b$ and a surface force $t$, one has to calculate $\left\|F-j^{*}(S)\right\|^{0}$ for any measure $S$. Using the expressions obtained above we have

$$
\begin{aligned}
\left(F-j^{*}(S)\right)(w)= & \int_{\operatorname{Int} B} w_{i} \mathrm{~d}\left(b_{i} \cdot V-s_{i 0}+s_{i m, m}\right) \\
& +\int_{\partial B} w_{i} \mathrm{~d}\left(t_{i} \cdot A-s_{i 0}+\operatorname{div}_{\partial} T_{i \partial}-s_{i m} v_{m}\right) .
\end{aligned}
$$

Thus,

$$
\begin{aligned}
\left\|F-j^{*}(S)\right\|^{0}=\sum_{i}\{ & \left|b_{i} \cdot V+s_{i m, m}-s_{i 0}\right|(\operatorname{Int} B) \\
& \left.+\left|t_{i} \cdot A-s_{i 0}+\operatorname{div}_{\partial} T_{i \partial}-s_{i m} v_{m}\right|(\partial B)\right\} .
\end{aligned}
$$

We conclude that

$$
\begin{aligned}
\|F\|=\inf \left\{\sum_{i}\right. & \left\{\left|b_{i} \cdot V+s_{i m, m}-s_{i 0}\right|(\operatorname{Int} B)\right. \\
& \left.\left.+\left|t_{i} \cdot A-s_{i 0}+\operatorname{div}_{\partial} T_{i \partial}-s_{i m} v_{m}\right|(\partial B)\right\}+\sum_{\alpha}\left|s_{\alpha}\right|(B)\right\}
\end{aligned}
$$

where the infimum is taken over all stress measures as defined above.

Alternatively, if we consider only stresses $S$ such that $F=j^{*}(S)$, then,

$$
\|F\|=\inf _{F=j^{*}(S)} \sum_{\alpha}\left|s_{\alpha}\right|(B) .
$$

3.10. Regularization of Stress Measures. In the expression of the flat norm it might be easier to evaluate the infimum over a dense subset. Specifically, we will try to evaluate it by using smooth mappings.

While measures on open sets may be regularized, i.e., may be approximated by smooth densities, the regions considered here require some additional attention because they include the boundaries. Radon measures may be approximated in the weak topology using a regularization process. The basic tool one uses is the Portmanteau theorem on weak convergence of measures (e.g., [2, p. 196], [3, p. 7]). Specifically, if the sequence of measures $\mu_{n}$ converges weakly to the measure $\mu$ and 
$D$ is a Borel set such that $\mu(\partial D)=0$, then, $\mu_{n}(D) \rightarrow \mu(D)$. In particular (see [7]), consider a Radon measure $\mu$ on an open set $\Omega \subset \mathbb{R}^{n}$ and a regularizing sequence $\mu_{n}$ of measures represented by smooth densities weakly approximating $\mu$. Then, if $D$ is an open subset whose closure is contained in $\Omega$ such that $|\mu|(\partial D)=0$, then, $|\mu|(D)=\lim _{\varepsilon \rightarrow 0}\left|\mu_{n}\right|(D)$. This we use in two situations.

Firstly, we consider the measure $\left.s_{i k}\right|_{\operatorname{Int} B}$ as a measure on an open set containing $B$. We have $\left.s\right|_{\operatorname{Int} B}(\partial B)=0$, so $s_{n} \rightarrow s$ weakly implies $\left|s_{n}\right|(\operatorname{Int} B) \rightarrow|s|(\operatorname{Int} B)$. Next, we consider a measure $T$ on $\partial B$. Since $\partial(\partial B)=\varnothing, T_{n} \rightarrow T$ weakly implies $T_{n}(\partial B) \rightarrow T(\partial B)$.

We recall that the subset containing distributions represented by smooth densities with compact supports is a dense subset (in the weak topology) of the space of all distributions on an open set. Hence, in particular we may approximate the measure $\left.s_{\alpha}\right|_{\operatorname{Int} B}$ on Int $B$ by smooth densities $\sigma_{\alpha \varepsilon}$ having compact support in Int $B$ such that

$$
\left.\int_{\operatorname{Int} B} \sigma_{\alpha \varepsilon} \phi \mathrm{d} V \longrightarrow \int_{\operatorname{Int} B} \phi \mathrm{d} s_{\alpha}\right|_{\operatorname{Int} B} \text { and } \int_{\operatorname{Int} B}\left|\sigma_{\alpha \varepsilon}\right| \mathrm{d} V \longrightarrow\left|s_{\alpha}\right|(\operatorname{Int} B)
$$

as $\varepsilon \rightarrow 0$. Similarly, as the measure $\left.s\right|_{\partial B}$ is a 0 -current on the manifold $\partial B$, it may be approximated by smooth densities $\tau_{\varepsilon}$ on $\partial B$ such that

$$
\left.\int_{\partial B} \tau_{\alpha \varepsilon} \phi \mathrm{d} A \longrightarrow \int_{\partial B} \phi \mathrm{d} s_{\alpha}\right|_{\partial B} \text { and } \int_{\partial B}\left|\tau_{\alpha \varepsilon}\right| \mathrm{d} A \longrightarrow\left|s_{\alpha}\right|(\partial B)
$$

as $\varepsilon \rightarrow 0$ (see de Rham [12, pp. 61-70]). In particular, we will use $\tau_{i \partial \varepsilon}$ to denote the densities approximating $T_{i \partial}$.

As regularization commutes with distributional derivatives and using the construction of the normal trace in [7] through a regularization process, any force $F$ in $C^{0}\left(B, \mathbb{R}^{3}\right)^{*} \cap C^{1}\left(B, \mathbb{R}^{3}\right)^{*}$ may be approximated in the form (we omit the $\varepsilon$ index)

$$
F(w)=\int_{\operatorname{Int} B}\left(\sigma_{i 0}-\sigma_{i m, m}\right) w_{i} \mathrm{~d} V+\int_{\partial B}\left(\tau_{i 0}-\operatorname{div}_{\partial} \tau_{i \partial}+\sigma_{i m} v_{m}\right) w_{i} \mathrm{~d} A .
$$

We conclude that the representation of a force in continuum mechanics by a body force and a surface force is natural in the sense that the elements of $C^{0}\left(B, \mathbb{R}^{3}\right)^{*} \cap$ $C^{1}\left(B, \mathbb{R}^{3}\right)^{*}$ may always be approximated this way.

We now turn to the evaluation of $\|F\|$ using smooth stress fields. Observing the expression for $\|F\|$, clearly $\left(b_{i}+\sigma_{i m \varepsilon, m}-\sigma_{i 0 \varepsilon}\right) \cdot V$ converges weakly to $\left(b_{i} \cdot V+\right.$ 
$\left.s_{i m, m}-s_{i 0}\right)\left.\right|_{\operatorname{Int} B}$ as $\varepsilon \rightarrow 0$ so

$$
\left|\left(b_{i}+\sigma_{i m \varepsilon, m}-\sigma_{i 0 \varepsilon}\right) \cdot V\right|(\operatorname{Int} B) \longrightarrow\left|b_{i} \cdot V+s_{i m, m}-s_{i 0}\right|(\operatorname{Int} B) .
$$

Similarly,

$$
\left(t_{i}-\tau_{i 0 \varepsilon}+\operatorname{div}_{\partial} \tau_{i \partial \varepsilon}-\sigma_{i m \varepsilon} v_{m}\right) \cdot A \longrightarrow t_{i} \cdot A-\left.s_{i 0}\right|_{\partial B}+\operatorname{div}_{\partial} T_{i \partial}-s_{i m} v_{m}
$$

weakly, so as $\varepsilon \rightarrow 0$,

$$
\left|\left(t_{i}-\tau_{i 0 \varepsilon}+\operatorname{div}_{\partial} \tau_{i \partial \varepsilon}-\sigma_{i m \varepsilon} v_{m}\right) \cdot A\right|(\partial B) \longrightarrow\left|t_{i} \cdot A-s_{i 0}+\operatorname{div}_{\partial} T_{i \partial}-s_{i m} v_{m}\right|(\partial B) .
$$

As the infimum may be obtained using the regularized smooth measures, it follows that

$$
\begin{aligned}
\|F\|=\inf \left\{\int_{\text {Int } B}(\right. & \left.\sum_{i}\left|b_{i}+\sigma_{i m, m}-\sigma_{i 0}\right|+\sum_{\alpha}\left|\sigma_{\alpha}\right|\right) \mathrm{d} V \\
& \left.+\int_{\partial B}\left(\sum_{i}\left|t_{i}-\tau_{i 0}+\operatorname{div}_{\partial} \tau_{i \partial}-\sigma_{i m} v_{m}\right|+\sum_{\alpha}\left|\tau_{\alpha}\right|\right) \mathrm{d} A,\right\}
\end{aligned}
$$

where the infimum is taken over all smooth $\sigma_{\alpha}$ over Int $B$ and $\tau_{\alpha}$ over $\partial B$.

If for the calculation of $\|F\|$ one uses only stresses that satisfy $f=j^{*}(S)$, i.e., $\left\|F-j^{*}(S)\right\|=0$. The basic representation theorem implies that for any $S$ that is in equilibrium with $F,\|F\| \leqslant\|S\|^{J}$ and specifically

$$
\|F\|=\inf \left\{\int_{\operatorname{Int} B} \sum_{\alpha}\left|\sigma_{\alpha}\right| \mathrm{d} V+\int_{\partial B} \sum_{\alpha}\left|\tau_{\alpha}\right| \mathrm{d} A\right\},
$$

where the infimum is taken over all smooth $\sigma_{\alpha}, \tau_{\alpha}$ satisfying

$$
\begin{aligned}
b_{i}+\sigma_{i m, m} & =\sigma_{i 0} & & \text { in Int } B, \\
\sigma_{i m} v_{m}+\tau_{i 0} & =t_{i}+\operatorname{div}_{\partial} \tau_{i \partial} & & \text { on } \partial B .
\end{aligned}
$$

Clearly, other methods (e.g., finite elements) may be utilized to approximate the stress measures. 
3.11. Representation without Self-Forces. We may use the representation of the flat norm as implied by Proposition 2.2 and Remark 2.3 for the case $q=1$. Such a representation will not include the self force terms $S_{0}$ given in terms of the measures $s_{0 i}$. The resulting expression for the flat norm of the force is

$$
\begin{aligned}
\|F\|=\inf \left\{\sum_{i}\right. & \left\{\left|b_{i} \cdot V+s_{i m, m}\right|(\operatorname{Int} B)\right. \\
& \left.\left.+\left|t_{i} \cdot A+\operatorname{div}_{\partial} T_{i \partial}-s_{i m} v_{m}\right|(\partial B)\right\}+\sum_{i, m}\left|s_{i m}\right|(B)\right\}
\end{aligned}
$$

and the expression using smooth stress fields is

$$
\begin{aligned}
\|F\|=\inf \left\{\int_{\operatorname{Int} B}\left(\sum_{i}\left|b_{i}+\sigma_{i m, m}\right|+\sum_{i, m}\left|\sigma_{i m}\right|\right) \mathrm{d} V\right. \\
\left.\quad+\int_{\partial B}\left(\sum_{i}\left|t_{i}+\operatorname{div}_{\partial} \tau_{i \partial}-\sigma_{i m} v_{m}\right|+\sum_{i, m}\left|\tau_{i m}\right|\right) \mathrm{d} A,\right\}
\end{aligned}
$$

over all smooth stress fields $\sigma_{i m}$ on Int $B$ and $\tau_{i m}$ on $\partial B$.

\section{SOBOLEV NORMS}

4.1. The $L^{p}$-Norms of the Force Functional. Using the same method we present additional estimates using the $L^{p}$-norms. Again, we regard the body as a measure space $B=\operatorname{Int} B \cup \partial B$ equipped with the Radon measure $\mu$ such that

$$
\mu(P)=V(P \cap \operatorname{Int} B)+A(P \cap \partial B) .
$$

For the initial seminorm on the space of differentiable virtual velocities we will use the $L_{(\mu)}^{p}$-seminorm, $1 \leqslant p<\infty$, relative to the Radon measure $\mu$ so

$$
\begin{aligned}
\|w\|^{0} & =\left(\int_{B} \sum_{i}\left|w_{i}\right|^{p} \mathrm{~d} \mu\right)^{1 / p} \\
& =\left(\int_{\operatorname{Int} B} \sum_{i}\left|w_{i}\right|^{p} \mathrm{~d} V+\int_{\partial B} \sum_{i}\left|w_{i}\right|^{p} \mathrm{~d} A\right)^{1 / p} .
\end{aligned}
$$


We consider force functionals $F$ that are linear and continuous relative to this seminorm. By the representation theorem of functionals on $L^{p}$ spaces, a force functional is represented by a field $f_{i}$ which is $L^{q}$ relative to $\mu$ with

$$
\frac{1}{p}+\frac{1}{q}=1
$$

in the form

$$
F(w)=\int_{B} f_{i} w_{i} \mathrm{~d} \mu=\int_{\operatorname{Int} B} b_{i} w_{i} \mathrm{~d} V+\int_{\partial B} t_{i} w_{i} \mathrm{~d} A .
$$

Here, $b_{i}=\left.f_{i}\right|_{\operatorname{Int} B}$ and $t_{i}=\left.f_{i}\right|_{\partial B}$ belong to $L^{q}(\operatorname{Int} B)$ and $L^{q}(\partial B)$, respectively. The dual norm $\|F\|^{0}$ of $F \in L_{(\mu)}^{q}\left(B, \mathbb{R}^{3}\right)$ is the $L_{(\mu)}^{q}$-norm

$$
\begin{aligned}
\|F\|^{0} & =\left(\int_{B} \sum_{i}\left|f_{i}\right|^{q} \mathrm{~d} \mu\right)^{1 / q} \\
& =\left(\int_{\operatorname{Int} B} \sum_{i}\left|b_{i}\right|^{q} \mathrm{~d} V+\int_{\partial B} \sum_{i}\left|t_{i}\right|^{q} \mathrm{~d} A\right)^{1 / q} .
\end{aligned}
$$

In particular, for $p=1$ the dual norm of $F$ is the $L^{\infty}$-seminorm so

$$
\|F\|^{0}=\operatorname{ess} \sup _{i, x \in B}\left|f_{i}(x)\right|=\max \left\{\operatorname{ess} \sup _{i, x \in B}\left|b_{i}(x)\right|, \text { ess } \sup _{i, x \in \partial B}\left|t_{i}(x)\right|\right\},
$$

where the essential supremum of $f$ is taken relative to the measure $\mu$ and those pertaining to $b$ and $t$ are taken relative to the volume and area measures, respectively.

4.2. The Representing Stresses. We wish to use the same mapping

$$
j: \mathbf{W}=C^{1}\left(B, \mathbb{R}^{3}\right) \longrightarrow J=C^{0}\left(B, \mathbb{R}^{12}\right)
$$

as in the previous section. In order that the general settings apply, it is required that $\|w\|=\|j(w)\|^{J} \geqslant\|w\|^{0}$ which puts a restriction on $\|\cdot\|^{J}$. The appropriate norm is suggested by the Sobolev embedding, or trace, theorem (see Maz'ja [11, p. 262], Adams [1, p. 216], Calderon [4]) implying that

$$
\left\|w\left|\partial B\left\|_{L^{p}} \leqslant A_{\partial}\right\| w\right|_{\operatorname{Int} B}\right\|_{L_{1}^{p}} .
$$

Here, $A_{\partial}$ is a constant depending only on $B$ that may be regarded as the norm of the trace mapping $\left.w\right|_{\operatorname{Int} B} \mapsto w \mid \partial B$. The seminorm $\|\cdot\|_{L_{1}^{p}}$ is the Sobolev seminorm 
in Int $B$ defined using the seminorm

$$
\|\psi\|_{L^{p}}=\left[\sum_{i, k} \int_{\operatorname{Int} B}\left|\psi_{i k}\right|^{p} \mathrm{~d} V\right]^{1 / p}, \quad k=0,1,2,3
$$

on $C^{0}\left(B, \mathbb{R}^{12}\right)$. Thus, $\|w\|_{L_{1}^{p}}=\|j(w)\|_{L^{p}}$, so

$$
\|w\|_{L_{p}^{1}}=\left[\sum_{i, k} \int_{\operatorname{Int} B}\left|w_{i, k}\right|^{p} \mathrm{~d} V\right]^{1 / p}
$$

Hence, the definition of the $\|\cdot\|^{0}$ seminorm implies that $\|w\|^{0} \leqslant A\|w\|_{L_{1}^{p}}$ for some positive constant $A$ depending only on $B$ and we assume that $A$ is the infimum of all such candidates. We set $\|\cdot\|^{J}=A\|\cdot\|_{L^{p}}$ so $\|w\|=A\|j(w)\|_{L^{p}}=A\|w\|_{L_{1}^{p}}$ and the basic requirement is satisfied.

Again, the representation theorem for $L^{p}$-functionals implies that every linear functional $S: J \rightarrow \mathbb{R}$ bounded by the $\|\cdot\|^{L^{p}}$-norm may be represented in the form

$$
S(\phi)=\int_{\operatorname{Int} B} \sigma_{i k} \phi_{i k} \mathrm{~d} V
$$

where $\sigma_{i k} \in L^{q}(B)$. In addition

$$
\|S\|_{L^{q}}=\left(\sum_{i, k}\left\|\sigma_{i k}\right\|_{L^{q}}^{q}\right)^{1 / q}=\left(\sum_{i, k} \int_{\operatorname{Int} B}\left|\sigma_{i k}\right|^{q} \mathrm{~d} V\right)^{1 / q} .
$$

Thus,

$$
\|S\|^{J}=\frac{1}{A}\left(\sum_{i, k}\left\|\sigma_{i k}\right\|_{L^{q}}^{q}\right)^{1 / q}=\frac{1}{A}\left(\sum_{i, k} \int_{\operatorname{Int} B}\left|\sigma_{i k}\right|^{q} \mathrm{~d} V\right)^{1 / q} .
$$

4.3. Representation of Forces by Stresses. Let a force $F \in L_{(\mu)}^{q}\left(B, \mathbb{R}^{3}\right)$ be represented by the $L^{q}$-stress fields $\sigma_{i k}, i=1,2,3, k=0,1,2,3$. Then,

$$
F(w)=S(j(w))=\int_{\operatorname{Int} B} w_{i} \sigma_{i 0} \mathrm{~d} V+\int_{\operatorname{Int} B} w_{i, m} \sigma_{i m} \mathrm{~d} V, \quad m=1,2,3 .
$$

The requirement that the force is in $L_{(\mu)}^{q}\left(B, \mathbb{R}^{3}\right)$ imposes regularity restriction on the stress components. The current $S_{1}$ is represented by the $L^{q}$ functions $\sigma_{i m}$ and its boundary $\partial S$, represented by the integral on the right, should be represented 
by $L_{(\mu)}^{q}$-fields. This is a particular case of the theory of divergence-measures of $L^{p}$-functions $[6,7]$ and denoting the divergence of $\sigma_{i m}$ by $\sigma_{i m, m}$ we may write

$$
F(w)=\int_{\operatorname{Int} B} w_{i} \sigma_{i 0} \mathrm{~d} V-\int_{\operatorname{Int} B} w_{i} \sigma_{i m, m} \mathrm{~d} V+\int_{\partial B} w_{i} \sigma_{i m} v_{m} \mathrm{~d} A
$$

If the force $F$ is given in terms of fields $b, t$, the condition $F=j^{*}(S)$ becomes

$$
\sigma_{i m, m}+b_{i}=\sigma_{i 0}, \quad t_{i}=\sigma_{i m} v_{m}
$$

4.4. The Sobolev norm of a force. Let $F \in L_{(\mu)}^{q}\left(B, \mathbb{R}^{3}\right)$ be given in terms of $b \in$ $L^{q}\left(\operatorname{Int} B, \mathbb{R}^{3}\right)$ and $t \in L^{q}\left(\partial B, \mathbb{R}^{3}\right)$. We wish to express the Sobolev norm of $F$ as a bound on the stress fields. The expression for $F-j^{*}(S)$ assumes the form

$$
\left(F-j^{*}(S)\right)(w)=\int_{\operatorname{Int} B}\left(b_{i}+\sigma_{i m, m}-\sigma_{i 0}\right) w_{i} \mathrm{~d} V+\int_{\partial B}\left(t_{i}-\sigma_{i m} v_{m}\right) w_{i} \mathrm{~d} A
$$

so $\left\|F-j^{*}(S)\right\|^{0}$ becomes

$$
\left\|F-j^{*}(S)\right\|^{0}=\left(\int_{\operatorname{Int} B} \sum_{i}\left|b_{i}+\sigma_{i m, m}-\sigma_{i 0}\right|^{q} \mathrm{~d} V+\int_{\partial B}\left|t_{i}-\sigma_{i m} v_{m}\right|^{q} \mathrm{~d} A\right)^{1 / q} .
$$

Thus,

$$
\begin{array}{r}
\|F\|=\inf \left\{\left(\int_{\operatorname{Int} B} \sum_{i}\left|b_{i}+\sigma_{i m, m}-\sigma_{i 0}\right|^{q} \mathrm{~d} V+\int_{\partial B}\left|t_{i}-\sigma_{i m} v_{m}\right|^{q} \mathrm{~d} A\right)^{1 / q}\right. \\
\left.+\frac{1}{A}\left(\int_{\operatorname{Int} B} \sum_{i, k}\left|\sigma_{i k}\right|^{q} \mathrm{~d} A\right)^{1 / q}\right\} .
\end{array}
$$

As before, we may approximate the stress components by smooth fields. Specifically (see [11, p. 11-14]), $C^{\infty}(B)$ is dense in $L^{q}(\operatorname{Int} B)$ and in the Sobolev spaces of functions on Int $B$. Hence, in the expression above we may assume that the functions $\sigma_{i k}$ are smooth on $B$.

The infimum is attained for some stress tensors $\hat{\sigma}_{i k}$ satisfying the equilibrium equations and boundary conditions so

$$
\|F\|=\frac{1}{A}\left(\sum_{i, k} \int_{\operatorname{Int} B}\left|\hat{\sigma}_{i k}\right|^{q} \mathrm{~d} V\right)^{1 / q} .
$$


Hence we conclude that

$$
\begin{aligned}
& \left(\sum_{i, k} \int_{\operatorname{Int} B}\left|\hat{\sigma}_{i k}\right|^{q} \mathrm{~d} V\right)^{1 / q} \\
& \quad=\inf \left\{A\left(\int_{\operatorname{Int} B} \sum_{i}\left|b_{i}-\sigma_{i 0}+\sigma_{i m, m}\right|^{q} \mathrm{~d} V+\int_{\partial B} \sum_{i}\left|t_{i}-\sigma_{i m} v_{m}\right|^{q} \mathrm{~d} A\right)^{1 / q}\right. \\
& \left.+\left(\sum_{i, k} \int_{B}\left|\sigma_{i k}\right|^{q} \mathrm{~d} V\right)^{1 / q}\right\} .
\end{aligned}
$$

As an example, consider the case where $p=1$ so that $q=\infty$. We recall that the $L^{\infty}$-seminorm is the essential supremum hence the last estimate implies that

$$
\text { ess } \sup _{i, k, x \in B}\left|\hat{\sigma}_{i k}\right|=\inf \left\{A \operatorname{ess} \sup _{i, x}\left\{\left|b_{i}-\sigma_{i 0}+\sigma_{i m, m}\right|,\left|t_{i}-\sigma_{i m} v_{m}\right|\right\}+\operatorname{ess} \sup _{i, k, x}\left|\sigma_{i k}\right|\right\} \text {, }
$$

where the infimum is taken over all smooth stress fields $\sigma_{i l}$ and self force fields $\sigma_{i 0}$.

4.5. Representation without Self-Forces. We consider the analog of Paragraph 3.11 using Proposition 2.2 and Remark 2.3 for the case of the $L^{p}$-seminorms. For the space $J^{0}$ we use $C^{0}\left(B, \mathbb{R}^{3}\right)$ and set $\left\|\psi_{0}\right\|^{J_{0}}=A\left\|\psi_{0}\right\|_{L^{p}}$. For the space $J^{1}$ we use $C^{0}\left(B, \mathbb{R}^{9}\right)$ with $\left\|\psi_{1}\right\|^{J_{0}}=A\left\|\psi_{1}\right\|_{L^{p}}$, where $A$ is defined in Paragraph 4.2. Thus,

$$
\|\psi\|^{J}=\left[\left(\left\|\psi_{0}\right\|^{J_{0}}\right)^{p}+\left(\left\|\psi_{1}\right\|^{J_{1}}\right)^{p}\right]^{1 / p}
$$

as required. The resulting expression for the Sobolev norm of the force does not contain the self force terms and

$$
\begin{array}{r}
\|F\|=\inf \left\{\left(\int_{\operatorname{Int} B} \sum_{i}\left|b_{i}+\sigma_{i m, m}\right|^{q} \mathrm{~d} V+\int_{\partial B}\left|t_{i}-\sigma_{i m} v_{m}\right|^{q} \mathrm{~d} A\right)^{1 / q}\right. \\
\left.+\frac{1}{A}\left(\int_{\operatorname{Int} B} \sum_{i, m}\left|\sigma_{i m}\right|^{q} \mathrm{~d} A\right)^{1 / q}\right\},
\end{array}
$$

where $m=1,2,3$ and the infimum is taken over all smooth tensor fields $\sigma_{i m}$.

\section{CONCLUDING REMARKS}

The foregoing material contains the definitions of various norms of force functionals. These norms may be approximated by minimization processes over classes of stress fields. Once evaluated, the theory states that there is some stress field $\hat{\sigma}$ 
that satisfies the equilibrium equation and boundary condition for the traction such that the norm of $\hat{\sigma}$ is equal to the norm of the force. All the above is completely independent of the material properties. In fact, the stress field $\hat{\sigma}$ is in general different then the actual stress field $\sigma_{a}$ for a particular material under the given loading. The norm of $\sigma_{a}$ is in general larger than that of $\hat{\sigma}$. Thus, $\|F\|=\|\hat{\sigma}\|^{J}$ provides us with an infimum for the norms of all actual stress fields.

The infimum provided by $\|F\|$ may be used as a guideline for the evaluation of structural optimization where one considers various distributions of material properties in the body. In other words, $\|F\|$ provides a lower bound for the norm $\left\|\sigma_{a}\right\|$ of the actual stress fields obtained for the candidate distributions of material properties.

Acknowledgements. The research leading to this paper was partially supported by the Paul Ivanier Center for Robotics Research and Production Management at BenGurion University.

\section{REFERENCES}

[1] R.A. Adams. Sobolev Spaces. Academic Press, New-York, 1975.

[2] R.B. Ash. Measure Integration and Functional Analysis. Academic Press, New-York, 1972.

[3] P. Billingsley. Convergence of Probability Measures. Wiley, New-York, 1999.

[4] A.P. Calderon. Lebesgue spaces of differentialbel functions and distributions. Proc. Symp. Pure Math., 4:33-49, 1961.

[5] G. Capriz. Continua with Microstructure. Springer, New York, 1989.

[6] G.-Q. Chen and H. Frid. Divergence-measure fields and hyperbolic conservations laws. Archive for Rational Mechanics and Analysis, 147:89-118, 1999.

[7] G.-Q. Chen and H. Frid. Extended divergence-measure fields and the euler equations for gas dynamics. Communications in Mathematical Physics, 236:251-280, 2003.

[8] H. Federer. Geometric Measure Theory. Springer Verlag, New York, 1969.

[9] M. Giaquinta, G. Modica and J. Souček. Cartesian Currents in the Calculus of Variations I. Springer, 1998.

[10] M.W. Hirsch. Differential Topology. Springer, 1976.

[11] V.G. Maz'ja. Sobolev Spaces. Springer, Berlin, 1985.

[12] G. de Rham. Differentiable Manifolds. Springer, Berlin, 1984.

[13] R. Segev. Forces and the existence of stresses in invariant continuum mechanics. Journal of Mathematical Physics, 27(1):163-170, 1986.

[14] R. Segev and G. deBotton. On the consistency conditions for force systems. International Journal of Nonlinear Mechanics, 26(1):47-59, 1991. 
[15] H. Whitney. Geometric Integration Theory. Princeton University Press, Princeton, New Jersey, 1957. [16] W.P. Ziemer. Weakly Differentiable Functions. Springer Verlag, New York, 1989.

Department of Mechanical Engineering, Ben-Gurion University, P.O.Box 653, BeerSHEVA 84105 ISRAEL

E-mail address: rsegev@bgumail.bgu.ac.il 